\title{
Desnutrição e fatores associados em crianças quilombolas menores de 60 meses em dois municípios do estado do Maranhão, Brasil
}

\author{
Malnutrition and associated factors among quilombola children \\ under 60 months of age in two cities of the state of Maranhão, \\ Brazil
}

Victor Nogueira da Cruz Silveira (https://orcid.org/0000-0002-5271-7412) ${ }^{1}$

Luana Lopes Padilha (https://orcid.org/0000-0002-7162-2726) ${ }^{2}$

Maria Tereza Borges Araújo Frota (https://orcid.org/0000-0001-6889-5785) ${ }^{1}$
${ }^{1}$ Departamento de Ciências Fisiológicas, Universidade Federal do Maranhão (UFMA). Av. Portugueses, Vila Bacanga. 65080-805 São Luís

MA Brasil.

victorncsilveira@gmail.com

${ }^{2}$ Programa de Pós-

Graduação em Saúde

Coletiva, Departamento de Saúde Pública, UFMA. São Luís MA Brasil.

\begin{abstract}
Objective: To evaluate the prevalence of malnutrition in children under 60 months of age living in quilombo remnant communities of two municipalities in the state of Maranhão and their associated factors. Methods: This is a cross-sectional study conducted with a sample of 372 children in August 2015. Socioeconomic, demograph$i c$, maternal and child information were collected. The anthropometry followed the one proposed by the World Health Organization. Height-for-age and weight-for-height rates were calculated using Anthro software version 3.2.2. Poisson regression was performed with a robust variance for the association of malnutrition with the studied variables, using Stata software version 14.0. Results: Child malnutrition was high for height-for-age (15.1\%) and weight-for-height (7\%). Children whose mothers had a short height $(<1.497 \mathrm{~m})$ were more likely to have height-for-age deficits ( $p$ $<0.05)$. No variables were statistically associated with low weight-for-height. Conclusion: Child malnutrition persists as a public health problem in vulnerable regions, and maternal factors such as low maternal height may explain the short stature of the children. The need for actions to address this nutritional deviation is pointed out.
\end{abstract}

Key words Preschoolers, Child malnutrition, Black people
Resumo $O$ objetivo deste artigo é avaliar a prevalência de desnutrição em crianças menores de 60 meses residentes em comunidades remanescentes de quilombos de dois municípios do estado do Maranhão e seus fatores associados. Estudo transversal com amostra de 372 crianças realizado em agosto de 2015. Foram coletadas informações socioeconômicas, demográficas, maternas e das crianças. A antropometria seguiu o proposto pela Organização Mundial da Saúde. Foram calculados os indices estatura para idade e peso para estatura utilizando o software Anthro versão 3.2.2. Foi realizada regressão de Poisson com variância robusta para a associação da desnutrição com as variáveis estudadas, utilizando o software Stata versão 14.0. A desnutrição em crianças foi elevada segundo a estatura-por-idade (15,1\%) e peso-para-estatura (7\%). Crianças cujas mães possuíam baixa estatura $(<1,497 \mathrm{~m})$ apresentaram mais chances de possuirem déficit de estatura-para-idade $(p<0,05)$. Nenhuma variável se associou estatisticamente ao baixo peso-para-estatura. A desnutrição infantil persiste como problema de saúde pública em regiões vulneráveis e os fatores maternos como a baixa estatura materna podem explicar a baixa estatura dos filhos. Aponta-se para a necessidade de ações de enfrentamento deste desvio nutricional.

Palavras-chave Pré-escolares, Desnutrição infantil, População negra 


\section{Introdução}

A população negra brasileira compreende $8,9 \%$ do contingente populacional do Brasil e está agrupada, especialmente, nas regiões Nordeste (11\%) e Sudeste $(9,7 \%)^{1}$. Parte significativa desta população se concentra nos estratos mais pobres do país, fruto de iniquidades sociais aos quais a raça negra historicamente foi submetida desde o período escravagista ${ }^{2,3}$.

Dentro da população negra geral existe um grupo social afro-brasileiro que descende de escravos e vive em áreas rurais isoladas, as quais, no período escravagista, serviram de resistência, e que mantêm fortes vínculos com a cultura negra africana, os quilombos ${ }^{3}$. Após o fim do período escravagista, essas comunidades passaram a ser designadas como remanescentes de quilombos ${ }^{2}$.

O Maranhão, um dos estados mais pobres do país, concentra a maior proporção de habitantes negros $(74 \%)^{1}$ e de comunidades remanescentes de quilombos ${ }^{4}$. Estas, por terem servido de refúgio, são isoladas, com pouca infraestrutura e difícil acesso a serviços básicos como saúde e educação ${ }^{5,6}$.

Em razão das consequências ocasionadas pela ancestralidade quilombola, as pessoas negras apresentam uma desigual inserção no mercado de trabalho, geralmente recorrendo a ocupações informais e consequentemente com rendimentos inferiores. O negro, quando comparado ao branco e ao amarelo, ganha em média 1,8 vezes menos ${ }^{7}$, o que reflete em suas condições de saúde e nutrição, especialmente em segmentos mais vulneráveis como mulheres e crianças menores de 60 meses.

Dentre os desvios nutricionais mais abrangentes nestes segmentos vulneráveis, está a desnutrição. Esta, na infância, resulta da interação de múltiplos fatores e suas consequências extrapolam o crescimento e o desenvolvimento adequado, afetando o desenvolvimento neurológico e, em casos extremos, resultando na morte precoce da criança ${ }^{5,6}$. A desnutrição é responsável por $30 \%$ dos óbitos de crianças menores de 60 meses, atingindo $50 \%$ nos países em desenvolvimento ${ }^{8}$.

No Brasil, dentre as causas de mortalidade infantil, a taxa por desnutrição ocupa a nona posição, figurando como uma das principais causas de óbito em todos os estados do Norte e Nordeste9. Concomitante a este cenário, ser da raça negra é a segunda maior causa de óbito infantil, quando se baseia na mortalidade por cor ou raça ${ }^{10}$.

Nas comunidades remanescentes de quilombos, pesquisas acerca do estado nutricional do público infantil são bastante escassas. Estudos desenvolvidos verificaram prevalência de desnutrição que variaram de 9,7\% em comunidades remanescentes de quilombos de Alagoas $^{5}$ a 31,1\% em comunidades do estado do Pará ${ }^{6}$ mostrando que a desnutrição infantil ainda figura como um dos problemas nutricionais importantes nas comunidades quilombolas.

Dessa forma, considerando a relevância de pesquisas dessa natureza, aliado ao fato de que conhecer este cenário nutricional de crianças quilombolas pode dar subsídio para o desenvolvimento de ações e estratégias voltadas à alimentação e nutrição destas comunidades, este estudo tem como objetivo avaliar a prevalência de desnutrição em crianças menores de 60 meses residentes em comunidades remanescentes de quilombos de dois municípios do estado do Maranhão e seus fatores associados.

\section{Método}

Trata-se de um estudo transversal integrado ao estudo "Condições de Nutrição e Segurança Alimentar de Mulheres e Crianças de Comunidades Quilombolas no Maranhão", desenvolvido com o objetivo de avaliar as condições de saúde e nutrição das crianças menores de cinco anos em comunidades quilombolas no Maranhão e que, além do estado nutricional, avaliou os aspectos socioeconômicos e demográficos, a situação de insegurança alimentar das famílias e o estado de anemia das mulheres e crianças.

A pesquisa foi realizada em comunidades remanescentes de quilombos dos municípios de Penalva e Viana, Maranhão, com ou sem título de posse coletiva da terra emitido por órgãos oficiais. Foram visitados todos os domicílios nos quais residia pelo menos uma criança menor de 60 meses em 27 comunidades localizadas na zona rural dos referidos municípios.

Os municípios foram selecionados por conveniência, por se tratarem de comunidades em que as lideranças locais foram receptivas à realização do estudo. A amostra foi do tipo probabilística, calculada com um nível de confiança de $95 \%$ e erro amostral de $5 \%$, tendo como referência o percentual de crianças com desnutrição na região Nordeste de 5,9\% ${ }^{11}$ e acrescentado $20 \%$ para eventuais perdas, totalizando 152 indivíduos.

A amostra do estudo foi de 373 crianças menores de 60 meses acompanhadas da mãe ou responsável em idade reprodutiva. Para o presente estudo foi excluída uma criança que se recusou 
a participar, perfazendo amostra total de 372 crianças. No caso de duas ou mais crianças no mesmo domicílio dentro da faixa etária do estudo, a mais nova era escolhida. Os critérios de não inclusão foram as crianças gêmeas e as portadoras de anemia falciforme referida.

A coleta de dados aconteceu em agosto de 2015, após contatos prévios com as lideranças locais para solicitar anuência e colaboração para a realização da pesquisa. Ao chegar ao município, essas lideranças intermediaram o contato com os Agentes Comunitários de Saúde (ACS) da Estratégia de Saúde da Família (ESF). A partir de então, eram elaborados os roteiros diários em cada comunidade, a identificação das famílias com o perfil desejado e a orientação das equipes para que procedessem a visita aos domicílios, sempre acompanhados do ACS responsável por aquela microárea e de um líder comunitário local.

O Termo de Consentimento Livre e Esclarecido (TCLE) era assinado após a concordância da participante em responder ao formulário. $\mathrm{O}$ original ficava com o entrevistador e uma cópia ficava no domicílio, em poder do entrevistado. A entrevistada que não assinava seu nome colocava a impressão digital no local da assinatura.

As entrevistas foram realizadas no domicílio da criança por meio de um formulário adaptado $^{12}$, validado e pré-codificado, aplicado prioritariamente com a mãe biológica e, na ausência desta, com a responsável ou cuidadora da criança, desde que habitasse no mesmo domicílio, que atendesse ao pré-requisito de idade e pudesse fornecer as informações solicitadas.

Paralelamente foram aferidas as medidas antropométricas de acordo com as recomendações da $\mathrm{WHO}^{13}$. O peso das crianças foi aferido utilizando como instrumento a balança marca Omron ${ }^{\circledR}$ (Omron Healthycare Inc U.S.A.), modelo HBF 214, com capacidade de $150 \mathrm{~kg}$ e graduações de $100 \mathrm{~g}$. As crianças menores de dois anos foram pesadas junto com a mãe com posterior cálculo de redução do peso ${ }^{13}$.

As crianças menores de dois anos tiveram seu comprimento medido em posição horizontal com infantômetro com amplitude de 100 $\mathrm{cm}$, marca Sanny ${ }^{\circledR}$. Para aferição da estatura das crianças maiores de dois anos foi utilizado o antropômetro de marca Alturexata ${ }^{\circledR}$, amplitude de 0 a 2,13 $\mathrm{m}$ e subdivisões de $0,1 \mathrm{~cm}$. Todos os indivíduos foram pesados e medidos sem sapatos, usando apenas roupas leves. Foram aferidas duas medidas de peso e altura e anotadas no formulário. A média de cada medida foi usada para a análise. Os entrevistadores trabalharam em du- plas na aferição das medidas antropométricas. Novas mensurações foram realizadas quando as duas medidas de peso diferiram em mais de 100 $\mathrm{g}$ e as de altura em mais $1,0 \mathrm{~cm}^{13}$.

As crianças foram avaliadas segundo os índices antropométricos peso para estatura $(\mathrm{P} / \mathrm{E})$ e estatura por idade (E/I) calculados em escore $\mathrm{Z}$ com auxílio do software Anthro versão 3.2.2 ${ }^{14}$ que se baseia na atual população de referência ${ }^{13}$. Foram estabelecidos os pontos de corte para avaliação do estado nutricional segundo referência da $\mathrm{WHO}^{13}$.

Ao final de cada dia de trabalho, os entrevistadores conferiam e codificavam os formulários, os quais eram revisados pelo supervisor de campo, visando detectar falhas de preenchimento que exigissem retorno imediato ao domicílio. Depois era preenchida a planilha de controle de entrevistas, para avaliar a cobertura da meta para aquela localidade e guardados os formulários.

Após a entrevista e colheita dos dados nutricionais, a equipe de campo orientou as famílias dos indivíduos estudados quanto à alimentação adequada e saudável. Os participantes que apresentaram risco nutricional e/ou resultado positivo para anemia foram encaminhados à Unidade Básica de Saúde daquela microárea.

A digitação dos dados foi realizada com dupla entrada, no programa Epi-info, versão 3.5.2, com o objetivo de identificar inconsistências na digitação. Para a análise dos dados, usou-se o programa STATA ${ }^{\circledR}$, versão 14.0 . Os fatores associados à desnutrição em crianças foram identificados em duas etapas. $\mathrm{Na}$ análise bivariada usou-se a regressão de Poisson simples para identificar as variáveis independentes que apresentaram associações com os déficits nutricionais ( $\mathrm{p}<0,20)$, as quais foram selecionadas para compor os modelos múltiplos. Na segunda etapa foram identificados os fatores associados aos déficits de estatura para idade e peso para estatura, mediante análise múltipla e hierarquizada de regressão de Poisson (erro padrão robusto), utilizando modelo conceitual adaptado ${ }^{15}$ (Figura 1):

- $1^{\circ}$ bloco - Fatores socioeconômicos e demográficos: sexo da criança e do chefe da família, idade da criança, escolaridade e vínculo trabalhista do chefe da família, renda familiar, total de pessoas na residência, participação no Programa Bolsa Família e Critério de Classificação Econômica Brasil;

- $2^{\circ}$ bloco - Acesso aos serviços e condições de habitação: Esgotamento sanitário, destino do lixo, procedência e tratamento da água;

- $3^{\circ}$ bloco - Fatores maternos: idade, aleitamento materno total e exclusivo, altura e IMC; 


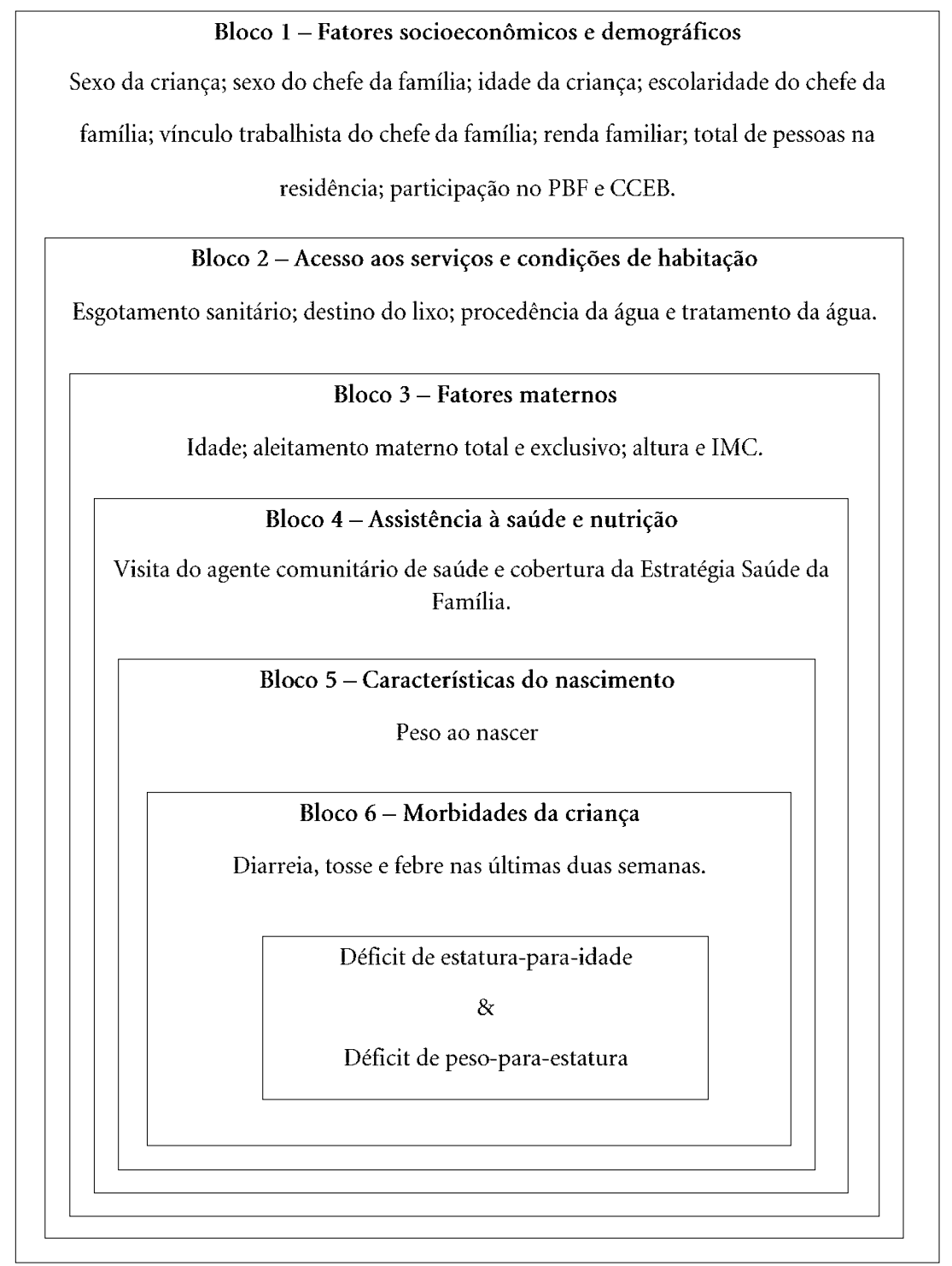

Figura 1. Modelo conceitual com seleção hierárquica para determinação de desnutrição em crianças menores de 60 meses.

Adaptado de Souza et al. ${ }^{15}$.

- $4^{\circ}$ bloco - Assistência à saúde e nutrição: Visita do agente comunitário de saúde e cobertura da Estratégia saúde da família;

- $5^{\circ}$ bloco - Características do nascimento: Peso ao nascer;

- 6o bloco - Morbidades da criança: Diarreia, tosse e febre nas últimas duas semanas
Iniciou-se a análise do modelo múltiplo do bloco distal para o proximal. As variáveis que apresentaram valor de $\mathrm{p}<0,20$ pela regressão de Poisson com variância robusta foram selecionadas como fator associado aos déficits nutricionais e permaneceram nos modelos múltiplos nas análises dos blocos subsequentes. No modelo final, 
foi considerada como variável estatisticamente significante aquela que apresentou p-valor $<0,05$ após todos os ajustes realizados.

O projeto foi aprovado pelo Comitê de Ética em Pesquisa da Universidade Federal do Maranhão, de acordo com os requisitos da Resolução do Conselho Nacional de Saúde (CNS 466/2012) e suas complementares.

\section{Resultados}

A média de idade das crianças estudadas foi de $23,1 \pm 15,7$ meses. Todas as características avaliadas estão descritas nas Tabelas 1 e 2. A prevalência de déficit de E/I foi de 15,1\% (IC95\%: 11,4 - 18,7) e de déficit de P/E foi de 7,0\% (IC95\%: $4,4-9,6)$.

Na Tabela 3 são apresentadas as análises bivariadas que associaram com o déficit de E/I. As variáveis associadas ao desfecho foram: a idade da criança, o sexo do chefe da família e a classe social do bloco socioeconômico e demográfico, $\mathrm{o}$ esgotamento sanitário do bloco de acesso aos serviços e condições de habitação, o tercil da altura materna do bloco de fatores maternos, o peso ao nascer do bloco de características do nascimento e a presença de diarreia e febre nas duas últimas semanas do bloco de morbidades da criança ( $\mathrm{p}$ $<0,20)$.

As variáveis associadas na análise bivariada seguiram para a análise múltipla e hierarquizada do déficit de E/I. O tercil da altura da mãe foi o único fator associado a esse desfecho (RP: 4,08; IC95\%: $1,95-8,52 ; p<0,001)$, evidenciando que o déficit desse índice foi mais prevalente em filhos de mulheres com altura inferior a $1,497 \mathrm{~m}$ (Tabela 3).

$\mathrm{Na}$ Tabela 4 estão descritas as análises bivariadas que associaram com o déficit de $\mathrm{P} / \mathrm{E}$, a saber: idade da criança do bloco socioeconômico e demográfico e; o tercil da altura e IMC da mãe do bloco de fatores maternos $(p<0,20)$. Nenhuma variável se associou com este desfecho na análise múltipla $(\mathrm{p}>0,05)$.

\section{Discussão}

Este estudo observou elevada prevalência de desnutrição, considerada de leve severidade para o déficit de $\mathrm{E} / \mathrm{I}$ e moderada para o déficit de $\mathrm{P} / \mathrm{E}^{16}$. Os fatores associados ao stunting foram a menor estatura materna e ao wasting, inicialmente, foram a idade da criança e a estatura materna.
Tabela 1. Distribuição das crianças quilombolas menores de cinco anos de idade segundo condições socioeconômicas e demográficas, acesso aos serviços e condições de habitação e saúde. Maranhão, 2015.

\begin{tabular}{|c|c|c|}
\hline Variáveis / Categorias & $\mathbf{n}$ & $\%$ \\
\hline \multicolumn{3}{|l|}{ Sexo da criança } \\
\hline Masculino & 182 & 48,92 \\
\hline Feminino & 190 & 51,08 \\
\hline
\end{tabular}

$\begin{array}{lrr}\text { Masculino } & 180 & 48,39 \\ \quad \text { Feminino } & 192 & 51,61 \\ \text { Escolaridade do chefe da família } & & \\ \quad 0-4 \text { anos } & 191 & 51,34 \\ >4 \text { anos } & 160 & 43,01 \\ \text { Sem informação } & 21 & 5,65\end{array}$

Vínculo trabalhista do chefe da

família

\begin{tabular}{|c|c|c|}
\hline Não trabalha & 74 & 19,89 \\
\hline Aposentado(a) & 15 & 4,03 \\
\hline Informal & 242 & 65,05 \\
\hline Formal & 40 & 10,75 \\
\hline Sem informação & 01 & 0,27 \\
\hline \multicolumn{3}{|l|}{ Renda familiar } \\
\hline$<1$ salário mínimo & 266 & 71,51 \\
\hline$\geq 1$ salário mínimo & 102 & 27,42 \\
\hline Sem informação & 04 & 1,08 \\
\hline \multicolumn{3}{|l|}{ Programa Bolsa Família } \\
\hline Sim & 307 & 85,53 \\
\hline Não & 07 & 1,88 \\
\hline Sem informação & 58 & 15,59 \\
\hline \multicolumn{3}{|l|}{ Critério de Classificação Econômica } \\
\hline \multicolumn{3}{|l|}{ Brasil } \\
\hline Classe C & 32 & 8,60 \\
\hline Classe D & 171 & 45,97 \\
\hline Classe E & 148 & 39,78 \\
\hline Sem informação & 21 & 5,65 \\
\hline \multicolumn{3}{|l|}{ Esgotamento sanitário } \\
\hline Rede pública / Fossa séptica & 42 & 11,35 \\
\hline $\begin{array}{l}\text { Fossa rudimentar / Vala / Céu } \\
\text { aberto }\end{array}$ & 328 & 88,65 \\
\hline Sem informação & 02 & 0,54 \\
\hline \multicolumn{3}{|l|}{ Procedência da água } \\
\hline Rede pública & 18 & 4,84 \\
\hline Poço / Cacimba / Barreiro / Cisterna & 354 & 95,16 \\
\hline \multicolumn{3}{|c|}{ Tratamento da água } \\
\hline Com tratamento & 178 & 47,85 \\
\hline Coada ou sem tratamento & 194 & 52,15 \\
\hline \multicolumn{3}{|l|}{ Total de pessoas na residência } \\
\hline $1-5$ pessoas & 242 & 65,05 \\
\hline 6 ou mais pessoas & 130 & 34,95 \\
\hline \multicolumn{3}{|l|}{ Destino do lixo } \\
\hline Coleta urbana & 01 & 0,27 \\
\hline Queimado / Enterrado & 347 & 93,28 \\
\hline Jogado a céu aberto & 24 & 6,45 \\
\hline Total & 372 & 100.00 \\
\hline
\end{tabular}


Tabela 2. Distribuição das crianças quilombolas menores de cinco anos de idade segundo fatores maternos e morbidade da criança. Maranhão, 2015.

\begin{tabular}{|c|c|c|}
\hline Variáveis / Categorias & $\mathbf{N}$ & $\%$ \\
\hline \multicolumn{3}{|l|}{ Idade da mãe } \\
\hline$>19$ anos & 319 & 85,75 \\
\hline$\leq 19$ anos & 53 & 14,25 \\
\hline \multicolumn{3}{|l|}{ Cor auto-referida da mãe } \\
\hline Branca & 16 & 4,30 \\
\hline Parda / Negra & 354 & 95,16 \\
\hline Sem informação & 02 & 0,54 \\
\hline \multicolumn{3}{|l|}{ Altura materna $(\mathrm{n}=248)$} \\
\hline $1^{\circ}(1.415-1.497 \mathrm{~m})$ & 127 & 34,14 \\
\hline $2^{\circ}(1.497-1.533 \mathrm{~m})$ & 121 & 32,53 \\
\hline $3^{\circ}(1.533-1.67 \mathrm{~m})$ & 124 & 33,33 \\
\hline \multicolumn{3}{|l|}{ IMC materno } \\
\hline Baixo peso & 20 & 5,38 \\
\hline Eutrofia & 191 & 51,34 \\
\hline Sobrepeso & 83 & 22,31 \\
\hline Obesidade & 49 & 13,17 \\
\hline Sem informação* & 29 & 7,80 \\
\hline \multicolumn{3}{|l|}{ Estratégia Saúde da Família } \\
\hline $\operatorname{Sim}$ & 60 & 16,13 \\
\hline Não & 309 & 83,06 \\
\hline Sem informação & 03 & 0,81 \\
\hline \multicolumn{3}{|l|}{$\begin{array}{l}\text { Visita do Agente Comunitário de } \\
\text { Saúde }\end{array}$} \\
\hline $\operatorname{Sim}$ & 334 & 89,78 \\
\hline Não & 38 & 10,22 \\
\hline \multicolumn{3}{|l|}{ Aleitamento materno total } \\
\hline Sim & 365 & 98,12 \\
\hline Não & 06 & 1,61 \\
\hline Sem informação & 01 & 0,27 \\
\hline \multicolumn{3}{|l|}{ Aleitamento materno exclusivo } \\
\hline $\begin{array}{l}\text { Não recebeu alimento antes dos } 6 \\
\text { meses }\end{array}$ & 228 & 61,29 \\
\hline Recebeu alimento antes dos 6 meses & 115 & 30,91 \\
\hline Sem informação & 29 & 7,80 \\
\hline \multicolumn{3}{|l|}{ Peso ao nascer $(\mathrm{n}=317)$} \\
\hline$<2500 \mathrm{~g}$ & 36 & 9,68 \\
\hline$\geq 2500 \mathrm{~g}$ & 281 & 75,54 \\
\hline Sem informação & 55 & 14,78 \\
\hline \multicolumn{3}{|l|}{ Diarreia nas últimas duas semanas } \\
\hline Sim & 327 & 87,90 \\
\hline Não & 45 & 12,10 \\
\hline \multicolumn{3}{|l|}{ Tosse } \\
\hline Sim & 190 & 51,08 \\
\hline Não & 182 & 48,92 \\
\hline Total & 372 & 100.00 \\
\hline
\end{tabular}

A alta prevalência de desnutrição aqui observada está relacionada com os fatores ambientais das famílias das crianças avaliadas, as quais, em sua maioria, estão inseridas em classes econômicas mais baixas e com menor poder aquisitivo.

Controversamente, a média de Escore- $Z$ do indicador $\mathrm{E} / \mathrm{I}(-0,74)$ das crianças avaliadas foi mais próxima do valor de adequação $(-2,0)$, quando comparada com a média nacional $(-1,32)$, entretanto a média do $\mathrm{P} / \mathrm{E}(-0,12)$ foi menor que a do país $(0,17)^{17}$.

O déficit de estatura-por-idade é comumente associado à privação alimentar a longo prazo $\mathrm{e}$ infecções frequentes ${ }^{5,6,17-21}$. Restrições no crescimento linear tendem a acontecer antes dos dois anos de idade com efeitos severos sobre o desenvolvimento psicomotor da criança, especialmente no rendimento escolar ${ }^{22-25}$. Entretanto, neste estudo, a prevalência de déficit estatural esteve uniformemente distribuída entre todas as faixas etárias das crianças.

A prevalência de déficit estatural encontrada ultrapassou a de outros estudos com crianças brasileiras com ou sem ancestralidade negra ${ }^{2,5,6,11,15,18-23,26}$. As maiores prevalências de stunting global pertencem à Ásia Meridional $(46 \%)$ e à África Subsaariana $(38 \%)^{25}$. O valor encontrado aqui se aproximou ao encontrado para a América Latina e Ásia Oriental $(16 \%)^{25}$, enquanto foi quase a metade da prevalência de países em desenvolvimento $(32 \%)^{25}$, em que o Brasil se classifica.

A prevalência de $15,1 \%$ de déficit estatural, observada neste estudo, corresponde a cinco vezes o esperado para a população de referência (2 $-3 \%)^{27}$, demonstrando o grau de risco nutricional ao qual as crianças quilombolas estão expostas. Observa-se que moradores de comunidades tradicionais como estas ainda convivem com limitadores na melhora de seu estado nutricional, como baixa renda e escolaridade, informalidade nos vínculos empregatícios e acesso dificultado a bens e serviços, fatores parcialmente superados pelo restante da população infantil brasileira ${ }^{1,7}$.

Neste estudo, a baixa estatura materna foi a única variável associada ao déficit estatural em crianças menores de 60 meses. Reconhecidamente, mulheres com alturas mais baixas tendem a gerar conceptos com comprimento igualmente baixo e provável crescimento limitado frente aos filhos de mulheres com altura normal ou alta $^{15,24,27}$. Esta relação pode estar associada a restrições no desenvolvimento e crescimento no útero materno, além de também perpetuar o ciclo da desnutrição crônica devido à manutenção da baixa estatura através das gerações subsequentes ${ }^{28}$. 
Tabela 3. Prevalência (\%) e razões de prevalência bruta e ajustada (RP) do déficit de estatura em crianças menores de 60 meses segundo fatores socioeconômicos e demográficos, acesso aos serviços e condições de habitação, fatores maternos, características do nascimento e morbidade da criança. Maranhão, 2015.

\begin{tabular}{|c|c|c|c|c|c|}
\hline \multirow{2}{*}{ Variáveis / Categorias } & \multicolumn{5}{|c|}{ Déficit de estatura para idade } \\
\hline & $\%$ & RP bruta & $\mathbf{p}$ & RP ajustada & $\mathbf{p}$ \\
\hline \multicolumn{6}{|c|}{ Fatores socioeconômicos e demográficos } \\
\hline \multicolumn{6}{|l|}{ Sexo da criança } \\
\hline Masculino & 50.0 & 0.95 & 0.844 & & \\
\hline Feminino & 50.0 & 1 & & & \\
\hline \multicolumn{6}{|l|}{ Idade da criança } \\
\hline$<24$ meses & 51.8 & 0.68 & 0.111 & 0.65 & 0.080 \\
\hline$\geq 24$ meses & 48.2 & 1 & & 1 & \\
\hline \multicolumn{6}{|l|}{ Sexo do chefe da família } \\
\hline Masculino & 39.3 & 1 & 0.150 & 1 & 0.276 \\
\hline Feminino & 60.7 & 1.44 & & 1.35 & \\
\hline \multicolumn{6}{|l|}{ Renda familiar } \\
\hline$<1$ salário mínimo & 73.2 & 1.05 & 0.866 & & \\
\hline$\geq 1$ salário mínimo & 26.8 & 1 & & & \\
\hline \multicolumn{6}{|l|}{ Classe social } \\
\hline Classe C & 1.9 & 0.16 & 0.071 & 0.20 & 0.115 \\
\hline Classe D & 45.3 & 0.73 & 0.231 & 0.66 & 0.101 \\
\hline Classe E & 52.8 & 1 & & 1 & \\
\hline \multicolumn{6}{|l|}{ Escolaridade da mãe } \\
\hline $0-4$ anos & 41.1 & 0.74 & 0.235 & & \\
\hline$>4$ anos & 58.9 & 1 & & & \\
\hline \multicolumn{6}{|c|}{ Acesso aos serviços e condições de habitação } \\
\hline \multicolumn{6}{|c|}{ Esgotamento sanitário } \\
\hline Rede pública/Fossa séptica & 3.6 & 1 & 0.077 & 1 & \\
\hline Fossa rudimentar/Vala & 96.4 & 3.46 & & 2.49 & 0.201 \\
\hline \multicolumn{6}{|l|}{ Abastecimento de água } \\
\hline Rede pública & 3.6 & 1 & 0.638 & & \\
\hline Poço/caçimba/barreiro/cisterna & 96.4 & 1.37 & & & \\
\hline \multicolumn{6}{|l|}{ Tratamento de água para beber } \\
\hline Com tratamento & 41.1 & 1 & 0.265 & & \\
\hline Coada ou sem tratamento & 58.9 & 1.37 & & & \\
\hline \multicolumn{6}{|l|}{ Total de pessoas no domicílio } \\
\hline $1-5$ pessoas & 60.7 & 1 & 0.432 & & \\
\hline 6 ou mais pessoas & 39.3 & 1.22 & & & \\
\hline
\end{tabular}

continua

Apesar da altura materna também ser conhecida por sua relação de causa - efeito com o baixo peso ao nascer, neste estudo não foi encontrado associação do peso ao nascimento com restrições no crescimento linear das crianças ${ }^{22,29,30}$.

O baixo peso-para-estatura é um dos indicadores mais fortes de mortalidade infantil para o grupo etário inferior a cinco anos de idade ${ }^{25}$. É comumente associado a formas mais agudas de privação alimentar ou episódios infecciosos ${ }^{25}$, 
Tabela 3. Prevalência (\%) e razões de prevalência bruta e ajustada (RP) do déficit de estatura em crianças menores de 60 meses segundo fatores socioeconômicos e demográficos, acesso aos serviços e condições de habitação, fatores maternos, características do nascimento e morbidade da criança. Maranhão, 2015.

\begin{tabular}{|c|c|c|c|c|c|}
\hline \multirow{2}{*}{ Variáveis / Categorias } & \multicolumn{5}{|c|}{ Déficit de estatura para idade } \\
\hline & $\%$ & RP bruta & p & RP ajustada & $\mathbf{p}$ \\
\hline \multicolumn{6}{|l|}{ Fatores maternos } \\
\hline \multicolumn{6}{|l|}{ Idade da mãe } \\
\hline$\leq 19$ anos & 8.9 & 0.59 & 0.217 & & \\
\hline$>19$ anos & 91.1 & 1 & & & \\
\hline \multicolumn{6}{|l|}{ Altura materna (tercil) } \\
\hline $1^{\circ}(1.415-1.497 \mathrm{~m})$ & 60.7 & 4.18 & $<0.001$ & 4.08 & $<0.001$ \\
\hline $2^{\circ}(1.497-1.533 \mathrm{~m})$ & 25.1 & 1.78 & & 1.73 & \\
\hline $3^{\circ}(1.533-1.67 \mathrm{~m})$ & 14.3 & 1 & & 1 & \\
\hline \multicolumn{6}{|l|}{ IMC da mãe } \\
\hline Baixo peso & 7.8 & 2.45 & 0.418 & & \\
\hline Eutrofia & 54.9 & 1.80 & & & \\
\hline Sobrepeso & 29.4 & 2.21 & & & \\
\hline Obesidade & 7.8 & 1 & & & \\
\hline \multicolumn{6}{|l|}{ Assistência à saúde e nutrição } \\
\hline \multicolumn{6}{|l|}{ Estratégia de Saúde na Família - ESF } \\
\hline Sim & 16.1 & 1 & 0.985 & & \\
\hline Não & 83.9 & 1.00 & & & \\
\hline \multicolumn{6}{|l|}{ Visita do Agente Comunitário de Saúde } \\
\hline $\operatorname{Sim}$ & 92.9 & 1 & 0.410 & & \\
\hline Não & 7.1 & 0.68 & & & \\
\hline \multicolumn{6}{|l|}{ Aleitamento materno } \\
\hline Sim & 98.2 & 1 & 0.898 & & \\
\hline Não & 1.8 & 1.13 & & & \\
\hline \multicolumn{6}{|l|}{ Aleitamento materno exclusivo } \\
\hline $\operatorname{Sim}$ & 66.7 & 1 & 0.974 & & \\
\hline Não & 33.3 & 1.00 & & & \\
\hline \multicolumn{6}{|l|}{ Características do nascimento } \\
\hline \multicolumn{6}{|l|}{ Peso ao nascer } \\
\hline$<2500 \mathrm{~g}$ & 18.2 & 1.74 & 0.124 & 0.94 & 0.937 \\
\hline$\geq 2500 \mathrm{~g}$ & 81.8 & 1 & & 1 & \\
\hline \multicolumn{6}{|l|}{ Morbidades da criança } \\
\hline \multicolumn{6}{|l|}{ Diarreia nas últimas duas semanas } \\
\hline Sim & 17.9 & 1.58 & 0.151 & 1.49 & 0.193 \\
\hline Não & 82.1 & 1 & & 1 & \\
\hline \multicolumn{6}{|l|}{ Tosse nas últimas duas semanas } \\
\hline $\operatorname{Sim}$ & 46.4 & 0.92 & 0.717 & & \\
\hline Não & 51 & 1 & & & \\
\hline \multicolumn{6}{|l|}{ Febre nas últimas duas semanas } \\
\hline Sim & 46.2 & 1.72 & 0.130 & 1.42 & 0.396 \\
\hline Não & 53.8 & 1 & & 1 & \\
\hline
\end{tabular}

$(7,8 \%)^{31}$. O valor encontrado neste estudo também se aproxima da prevalência observada em países em desenvolvimento $(7,7 \%)^{31}$. No presente estudo, o déficit encontrado também foi superior ao esperado para a população de referência (2 $3 \%)^{27}$, sendo mais que o dobro do limite superior.
O peso-por-estatura se mostrou homogêneo quando distribuído pelo sexo das crianças, entretanto, ao classificar pela idade, se observou que crianças com menos de 24 meses possuíam maior propensão em desenvolver baixo peso, corroborando com dados da literatura que in- 
Tabela 4. Prevalência (\%) e razões de prevalência bruta e ajustada (RP) do déficit de peso para estatura em crianças menores de 60 meses segundo fatores socioeconômicos e demográficos, acesso aos serviços e condições de habitação, fatores maternos, características do nascimento e morbidade da criança. Maranhão, 2015.

\begin{tabular}{|c|c|c|c|c|c|}
\hline \multirow{2}{*}{ Variáveis / Categorias } & \multicolumn{5}{|c|}{ Déficit de peso para estatura } \\
\hline & $\%$ & RP bruta & $\mathrm{p}$ & RP ajustada & $\mathbf{p}$ \\
\hline \multicolumn{6}{|c|}{ Fatores socioeconômicos e demográficos } \\
\hline \multicolumn{6}{|c|}{ Sexo da criança } \\
\hline Masculino & 53.9 & 0.81 & 0.594 & & \\
\hline Feminino & 46.1 & 1 & & & \\
\hline \multicolumn{6}{|l|}{ Idade da criança } \\
\hline$<24$ meses & 23.1 & 1 & & 1 & \\
\hline$\geq 24$ meses & 76.9 & 2.41 & 0.052 & 2.34 & 0.090 \\
\hline \multicolumn{6}{|l|}{ Sexo do chefe da família } \\
\hline Masculino & 50 & 1 & & & \\
\hline Feminino & 50 & 0.93 & 0.853 & & \\
\hline \multicolumn{6}{|l|}{ Renda familiar } \\
\hline$<1$ salário mínimo & 76.9 & 1.28 & 0.581 & & \\
\hline$\geq 1$ salário mínimo & 23.1 & 1 & & & \\
\hline \multicolumn{6}{|l|}{ Classe social } \\
\hline Classe C & 3.8 & 0.35 & 0.308 & & \\
\hline Classe D & 46.2 & 0.79 & 0.548 & & \\
\hline Classe E & 50 & 1 & & & \\
\hline \multicolumn{6}{|l|}{ Escolaridade da mãe } \\
\hline$\leq 4$ anos & 53.8 & 0.91 & 0.825 & & \\
\hline$>4$ anos & 46.2 & 1 & & & \\
\hline \multicolumn{6}{|c|}{ Acesso aos serviços e condições de habitação } \\
\hline \multicolumn{6}{|c|}{ Esgotamento sanitário } \\
\hline Rede pública/Fossa séptica & 3.8 & 1 & & & \\
\hline Fossa rudimentar/Vala & 96.2 & 3.2 & 0.247 & & \\
\hline \multicolumn{6}{|l|}{ Abastecimento de água } \\
\hline Rede pública & 3.8 & 1 & 0.807 & & \\
\hline Poço/caçimba/barreiro/cisterna & 96.2 & 1.27 & & & \\
\hline \multicolumn{6}{|l|}{ Tratamento de água para beber } \\
\hline Com tratamento & 46.2 & 1 & & & \\
\hline Coada ou sem tratamento & 53.8 & 1.07 & 0.847 & & \\
\hline \multicolumn{6}{|l|}{ Total de pessoas no domicílio } \\
\hline $1-5$ pessoas & 57.7 & 1 & & & \\
\hline 6 ou mais pessoas & 42.3 & 1.38 & 0.398 & & \\
\hline
\end{tabular}

continua

dicam a população de menores de dois anos de idade como a mais afetada no retardo do ganho de peso $^{5,32,33}$.

O emaciamento causado pelo déficit neste indicador retrata uma ruptura na harmonia corporal da criança ${ }^{13,15}$, possivelmente causada por ganho de peso inadequado, apesar de crescimento normal, ou perda de peso momentânea devido a morbidades comuns na infância, como tosse e diarrei ${ }^{34}$. Este estudo, no entanto, não encontrou associação de episódios de morbidade com o déficit neste indicador, provavelmente atribuindo ao déficit de P/E o ganho de peso inadequado, o qual não pôde ser avaliado devido à natureza transversal do estudo.

As prevalências de déficit de peso e estatura sugerem que as crianças quilombolas menores de 60 meses de Penalva e Viana ainda convivam com a inacessibilidade a serviços essenciais. As prevalências mais próximas às encontradas neste estudo foram em regiões geográficas mais pobres, principalmente no continente africano, de onde são oriundos os ancestrais dos remanescentes de quilombos $2,5,6,11,15,18-23,25,26,31,32$. 
Tabela 4. Prevalência (\%) e razões de prevalência bruta e ajustada (RP) do déficit de peso para estatura em crianças menores de 60 meses segundo fatores socioeconômicos e demográficos, acesso aos serviços e condições de habitação, fatores maternos, características do nascimento e morbidade da criança. Maranhão, 2015.

\begin{tabular}{|c|c|c|c|c|c|}
\hline \multirow{2}{*}{ Variáveis / Categorias } & \multicolumn{5}{|c|}{ Déficit de peso para estatura } \\
\hline & $\%$ & RP bruta & $\mathbf{p}$ & RP ajustada & $\mathbf{p}$ \\
\hline \multicolumn{6}{|l|}{ Fatores maternos } \\
\hline \multicolumn{6}{|l|}{ Idade da mãe } \\
\hline$\leq 19$ anos & 88.5 & 0.78 & 0.681 & & \\
\hline$>19$ anos & 11.5 & 1 & & & \\
\hline \multicolumn{6}{|l|}{ Altura materna (tercil) } \\
\hline $1^{\circ}(1.415-1.497 \mathrm{~m})$ & 53.9 & 1.39 & 0.045 & 2.33 & 0.102 \\
\hline $2^{\circ}(1.497-1.533 \mathrm{~m})$ & 26.9 & 0.82 & 0.528 & 0.78 & 0.707 \\
\hline $3^{\circ}(1.533-1.67 \mathrm{~m})$ & 19.2 & 1 & & 1 & \\
\hline \multicolumn{6}{|l|}{ IMC da mãe } \\
\hline Baixo peso & 18.2 & 2.45 & 0.172 & 1.61 & 0.469 \\
\hline Eutrofia & 50 & 0.71 & 0.541 & 0.64 & 0.438 \\
\hline Sobrepeso & 13.6 & 0.44 & 0.273 & 0.39 & 0.195 \\
\hline Obesidade & 18.2 & 1 & & 1 & \\
\hline \multicolumn{6}{|l|}{ Assistência à saúde e nutrição } \\
\hline \multicolumn{6}{|l|}{ Estratégia de Saúde na Família - ESF } \\
\hline Sim & 24 & 1 & & & \\
\hline Não & 76 & 0.60 & 0.260 & & \\
\hline \multicolumn{6}{|l|}{ Visita do Agente Comunitário de Saúde } \\
\hline Sim & 88.4 & 1 & & & \\
\hline Não & 11.6 & 1.14 & 0.821 & & \\
\hline \multicolumn{6}{|l|}{ Aleitamento materno exclusivo } \\
\hline Sim & 66.7 & 1 & & & \\
\hline Não & 33.3 & 0.98 & 0.975 & & \\
\hline \multicolumn{6}{|l|}{ Características do nascimento } \\
\hline \multicolumn{6}{|l|}{ Peso ao nascer } \\
\hline$<2500 \mathrm{~g}$ & 15 & 1.37 & 0.599 & & \\
\hline$\geq 2500 \mathrm{~g}$ & 85 & 1 & & & \\
\hline \multicolumn{6}{|l|}{ Morbidades da criança } \\
\hline \multicolumn{6}{|l|}{ Diarreia nas últimas duas semanas } \\
\hline Sim & 19.2 & 1 & & & \\
\hline Não & 80.8 & 1.72 & 0.248 & & \\
\hline \multicolumn{6}{|l|}{ Tosse nas últimas duas semanas } \\
\hline Sim & 42.3 & 1 & & & \\
\hline Não & 57.7 & 0.77 & 0.495 & & \\
\hline \multicolumn{6}{|l|}{ Febre nas últimas duas semanas } \\
\hline $\operatorname{Sim}$ & 27.3 & 1 & & & \\
\hline Não & 72.7 & 0.75 & 0.672 & & \\
\hline
\end{tabular}

Este trabalho teve por limitação em seu delineamento o levantamento de dados em dois municípios de mesma região geográfica, sem levar em consideração a distribuição da população quilombola nos outros municípios no Maranhão, o que não permite inferir estes resultados para todas as comunidades quilombolas do estado. Além disso, a ausência da avaliação do consumo alimentar desta população restringiu a verificação de inadequações na dieta das crianças, as quais poderiam explicar a desnutrição encontrada.

O levantamento de informações em uma amostra representativa nas comunidades remanescentes de quilombos do estado do Maranhão constitui iniciativa pioneira de analisar a situação nutricional desta população, o que pode aperfeiçoar a compreensão da desnutrição em populações 
específicas em um dos estados mais pobres do país e, portanto, direcionar incrementos nas políticas públicas para este grupo populacional específico.

\section{Conclusão}

O presente estudo observou elevadas prevalências de déficit estatural e de baixo peso-para-es- tatura na amostra de crianças quilombolas em dois municípios no estado do Maranhão. A menor estatura materna se relacionou com o déficit de estatura infantil. Os resultados encontrados expõem a situação de iniquidade social e invisibilidade a que essa população está submetida e apontam para a necessidade de políticas públicas que visem compensar a negligência histórica do Estado para com esse segmento populacional.

\section{Colaboradores}

VNC Silveira trabalhou na interpretação dos dados redação do artigo e aprovação da versão a ser publicada. MTBA Frota trabalhou na concepção, revisão crítica do trabalho e aprovação da versão a ser publicada. LL Padilha trabalhou na análise dos dados, revisão crítica do trabalho e aprovação da versão a ser publicada.

\section{Agradecimentos}

Agradecemos à Secretaria de Estado da Saúde do Maranhão e à Secretaria de Políticas de Promoção da Igualdade Racial pelo apoio logístico e financeiro. Agradecemos também à Associação Santo Antônio dos Pretos pelo apoio e permissão à pesquisa. Obrigado também a todos que direta ou indiretamente tenham contribuído para a realização deste trabalho.

\section{Referências}

1. Instituto Brasileiro de Geografia e Estatística (IBGE). Pesquisa Nacional por Amostra de Domicílios. Rio de Janeiro: IBGE; 2016.

2. Brasil. Ministério do Desenvolvimento Social e Combate à Fome (MDS). Cadernos de Estudos: Quilombos do Brasil: Segurança alimentar e nutricional em territórios titulados. Brasília: MDS; 2014.

3. Lindoso D. A razão quilombola: estudos em torno do conceito quilombola de nação etnográfica. Maceió: EDUFAL; 2011.

4. Brasil. Ministério do Desenvolvimento Social e Combate à Fome (MDS). Guia de Cadastramento de Famílias Quilombolas. Brasília: SENARC/MDS; 2015.

5. Leite FMB, Ferreira HS, Bezerra MKA, Assunção ML, Horta BL. Consumo alimentar e estado nutricional de pré-escolares das comunidades remanescentes de quilombos do estado de alagoas. Rev Paul Pediatr 2013; 31(4):444-451.

6. Guimarães RCR, Silva HP. Estado nutricional e crescimento de crianças quilombolas de diferentes comunidades do estado do Pará. Amazonica 2015; 7(1):186209.

7. Instituto Brasileiro de Geografia e Estatística (IBGE). Pesquisa Nacional por Amostra de Domicílios Contínua. Brasília: IBGE; 2017.

8. Frempong RB, Annim SK. Dietary diversity and child malnutrition in Ghana. Heliyon 2017; 3(5):1-21.

9. França EB, Lansky S, Rego MAS, Malta DC, França JS, Teixeira R, Porto D, Almeida MF, Souza MFM, Szwarcwald CL, Mooney M, Naghavi M, Vasconcelos AMN. Principais causas da mortalidade na infância no Brasil, em 1990 e 2015: estimativas do estudo de Carga Global de Doença. Rev Bras Epidemiol 2017; 20(1S):46-60. 
10. Caldas ADR, Santos RV, Borges GM, Valente JG, Portela MC, Marinho GL. Mortalidade infantil segundo cor ou raça com base no Censo Demográfico de 2010 e nos sistemas nacionais de informação em saúde no Brasil. Cad Saude Publica 2017; 33(7):1-13.

11. Brasil. Ministério da Saúde (MS). Pesquisa Nacional de Demografia e Saúde - 2006 (PNDS 2006): Dimensões do Processo Reprodutivo e da Saúde da Criança. Brasília: MS; 2009.

12. Universidade Federal do Pernambuco (UFPE). III Pesquisa Estadual de Saúde e Nutrição: Saúde, Nutrição, Alimentação, Condições Socioeconômicas e Atenção à Saúde no Estado de Pernambuco. Recife: UFPE; 2010.

13. World Health Organization (WHO). Child growth standards: length/height-for-age, weight-for-age, weight-for-length, weight-for-height and body mass indexfor-age: methods and development. Geneva: WHO; 2006.

14. World Health Organization (WHO). Child growth standards: WHO Anthro (versão 3.2.2.) e macros. Geneva: WHO; 2011.

15. Souza OF, D’Aquino Benício MH, Castro TG, Muniz PT, Cardoso MA. Desnutrição em crianças menores de 60 meses em dois municípios no estado do Acre: Prevalência e fatores associados. Rev Bras Epidemiol 2012; 15(1):211-221.

16. World Health Organization (WHO). WHO global database on child growth and malnutrition. Geneva: WHO; 1997.

17. Instituto Brasileiro de Geografia e Estatística (IBGE). Antropometria e análise do estado nutricional de crianças e adolescentes no Brasil. Brasília: IBGE; 2006.

18. Barroso MMB, Salvador LM, Neto UF. Desnutrição proteico-calórica grave em dois irmãos devido ao abuso por privação alimentar. Rev Paul Pediatr 2016; 34(4):522-527.

19. Pedraza DF, Oliveira MM, Cardoso MVLML, Araujo EMN, Rocha ACD. Índices antropométricos de crianças assistidas em creches e sua relação com fatores socioeconômicos, maternos e infantis. Cien Saude Colet 2016; 21(7):2219-2232.

20. Pedraza DF, Rocha ACD, Sousa CPC. Crescimento e deficiência de micronutrientes: perfil das crianças assistidas no núcleo de creches do governo da Paraíba, Brasil. Cien Saude Colet 2013; 18(11):3379-3390.

21. Pedraza DF, Silva FA, Melo NLS, Araujo EMN, Sousa CPC. Estado nutricional e hábitos alimentares de escolares de Campina Grande, Paraíba, Brasil. Cien Saude Colet 2017; 22(2):469-477.

22. Vargas CL, Benedetti FJ, Weinmann ARM. Crescimento de prematuros até os dois anos de vida: revisão integrativa da literatura. Rev Bras Crescimento desenvolv Hum 2017; 3(1):72-84.

23. Pereira IFS, Andrade LMB, Spyrides MHC, Lyra CO. Estado nutricional de menores de 5 anos de idade no Brasil: evidências da polarização epidemiológica nutricional. Cien Saude Colet 2017; 22 (10):3341-3352.
24. Araújo TS, Oliveira CSM, Muniz PT, Silva-Nunes M, Cardoso MA. Desnutrição infantil em um dos municípios de maior risco nutricional do Brasil: estudo de base populacional na Amazônia Ocidental Brasileira. Rev Bras Epidemiol 2016; 19(3):554-566.

25. United Nations Children's Fund (UNICEF). Progress for Children: A World Fit for Children Statistical Review (no6). New York: UNICEF; 2007.

26. Brasil. Ministério do Desenvolvimento Social e Combate à Fome (MDS). Chamada Nutricional Quilombola. Brasília: SEPPIR/MDS; 2007.

27. Monteiro CA, Onis M, Clugston G. The worldwide magnitude of protein-energy malnutrition: an overview from the WHO Global Database on Child Growth. Bulletin of the World Health Organization 1993; 71(6):703-712.

28. Black RE, Victora CG, Walker SP, Bhutta ZA, Christian P, Onis M, Ezzati M, Grantham McGregor S, Katz J, Martorell R, Uauy R. Maternal and child undernutrition and overweight in low-income and middle-income countries. Lancet Child Adolesc Health 2013; 382(9890):427-451.

29. Pedraza DF, Souza MM, Cristóvão FS, França ISX. Baixo peso ao nascer no Brasil: revisão sistemática de estudos baseados no Sistema de Informações sobre Nascidos Vivos. Pediatria Moderna 2014; 50(2):51-64.

30. Gonçalves FCLSP, Lira PIC, Eickmann SH, Lima MC. Razão peso/perímetro cefálico ao nascer na avaliação do crescimento fetal. Cad Saude Publica 2015; 31(9):1995-2004.

31. International Bank for Reconstruction and Development (IBRD). Prevalence of wasting, weight for height (\% of children under 5). Washington: IBRD; 2016.

32. Pedraza DF, Menezes TN. Caracterização dos estudos de avaliação antropométrica de crianças brasileiras assistidas em creches. Rev Paul Pediatr 2016; 34(2):216-224.

33. Pedraza DF, Sales MC, Menezes TN. Fatores associados ao crescimento linear de crianças socialmente vulneráveis do Estado da Paraíba, Brasil. Cien e Saude Colet 2016; 21(3):935-945.

34. Pedraza DF. Hospitalização por doenças infecciosas, parasitismo e evolução nutricional de crianças atendidas em creches públicas. Cien e Saude Colet 2017; 22(12):4105-4114.

Artigo apresentado em 15/11/2017

Aprovado em 25/09/2018

Versão final apresentada em 27/09/2018 